\title{
Scaling of the von Neumann entropy across a finite temperature phase transition
}

\author{
Vladislav Popkov and Mario Salerno \\ Dipartimento di Fisica "E.R. Caianiello" and Consorzio Nazionale \\ Interuniversitario per le Scienze Fisiche della Materia (CNISM), \\ Universitá di Salerno, I-84081, Baronissi (SA), Italy
}

\begin{abstract}
The spectrum of the reduced density matrix and the temperature dependence of the von Neumann entropy (VNE) are analytically obtained for a system of hard core bosons on a complete graph which exhibits a phase transition to a Bose-Einstein condensate at $T=T_{c}$. It is demonstrated that the VNE undergoes a crossover from purely logarithmic at $T=0$ to purely linear in block size $n$ behaviour for $T \geq T_{c}$. For intermediate temperatures, VNE is a sum of two contributions which are identified as the classical (Gibbs) and the quantum (due to entanglement) parts of the von Neumann entropy.
\end{abstract}

PACS numbers: 03.67.Mn, 03.75.Gg, 87.15.Zg

Introduction. Recent experimental progresses in quantum communication have increased interest on entanglement properties of quantum systems both as resource for quantum computing and as intrinsic characterization of quantum states [1, 2]. A key concept to quantify the entanglement of a part of a system (e.g. a system of qubits) with respect to the rest (environment) is the von Neumann entropy (VNE) which has been shown to be related to the maximal compression rate of a quantum information in an ideal coding scheme for two subsystems in a joint pure state [2]. In the case of mixed states the VNE contains contributions which come both from classical and quantum (entanglement) correlations. In order to extract the entanglement part from the VNE the classical contribution must be subtracted, this requiring additional minimization over all possible disentangled states (see, e.g., [3, 4]). The computational time needed to perform such a minimization grows exponentially with the size of the system, making the calculations impossible for any system with more than a few particles.

In exceptional cases, however, the von Neumann entropy can be computed exactly due to symmetries of the underlying quantum problem. An example of this is given by integrable critical spin chains which have ground states exactly known in the thermodynamic limit, with long-distance correlations governed by conformal field theory [5]. In this case VNE has been proved [6, 7] to behave universally as $S_{(n)}=\frac{c}{3} \log \left(\frac{1}{\pi T} \sinh (\pi n T)\right)+$ const, in the limit of a large block size $n$ and as a function of the temperature $T$, where $c$ is the central charge of the underlying conformal field theory.

In the present letter, we calculate, both analytically and numerically, the dependence on the temperature of the von Neumann entropy for a class of quantum models which are invariant under the action of the symmetric group and which arise in several physical contexts, see e.g. [8, 9, 10, 11, 12, 13, 14]. A common physical property of these models is that they exhibit a phase transition from an ordered to a disordered state at a finite critical temperature $T_{c}$ [10]. The knowledge of the ex- act analytical expression of the VNE together with the requirement that the classical part of the entropy must behave extensively for large $n$, allow us to single out the entanglement part of the VNE for arbitrary $n$ and $T$. As a result, we show that the extensive part of the VNE coincides with the Gibbs entropy computed directly from the energy spectrum, while the entaglement part scales as $S_{(n)}-S_{\text {Gibbs }}=\frac{1}{2} \log n+$ const below $T_{c}$ and as $S_{(n)}=S_{\text {Gibbs }}$ above $T_{c}$. For $T=0$ the extensive part $S_{\text {Gibbs }}$ disappears and the result in Ref. [15] is recovered.

The model. As physical model we consider a system of hard-core bosons described by the Hamiltonian

$$
H=-\frac{1}{L} \sum_{i, j}^{L} b_{i}^{+} b_{j}+\sum_{i=1}^{L} b_{i}^{+} b_{i},
$$

where $b_{i}^{\dagger}, b_{i}$, denote creation and annihilation operators satisfying the following hard-core Heisenberg algebra: $\left[b_{i}, b_{j}\right]=\left[b_{i}^{+}, b_{j}^{+}\right]=0,\left[b_{i}, b_{j}^{+}\right]=\left(1-2 b_{j}^{+} b_{i}\right) \delta_{i j}$. Due to the on-site Fermi-like commutation relations, double occupancy is not allowed: the action of $b_{i}^{+}$and $b_{i}$ on the single particle Fock space being $b_{i}^{+}|0\rangle=|1\rangle ; \quad b_{i}|1\rangle=|0\rangle$; $b_{i}|0\rangle=b_{i}^{+}|1\rangle=0$. Note that the Hamiltonian (11) is invariant under the action of the permutation group (symmetric group $S_{L}$ ) and conserves the number of particles $N=\sum_{i=1}^{L} b_{i}^{+} b_{i}$ with $N \leq L$. The ground state of the system in the sector with $N$ particles is given by the symmetric state $|\Psi(L, N)\rangle=\left(\begin{array}{c}L \\ N\end{array}\right)^{-1 / 2} \sum_{P}|1111111 \ldots 000000\rangle$ where sum is taken over all possible distributions of $N$ particles among $L$ sites. Note that symmetric states are intensively studied with symmetry techniques for testing various entanglement measures, see e.g. 16] and references therein. The excited states can be constructed from the irreducible representations (irreps) of $S_{L}$ using filled Young tableau (YT) of type $\{L-r, r\}$ with $r$ assuming all values in the interval $[L / 2] \geq r \geq 1$ where $[x]$ denotes the integer part of $x$ (see [12, 17]). The spectral and thermodynamical properties of the system were studied in 12 where it was shown that eigenvalues of (11) associated to 
YTs of type $\{L-r, r\}$ are given by

$$
E_{r}=r+\frac{1}{L}(N(N-1)-r(r-1)), \quad r=0,1, \ldots, N,
$$

with degeneracy $d_{r}=\left(\begin{array}{c}L \\ r\end{array}\right)-\left(\begin{array}{c}L \\ r-1\end{array}\right)$ [12]. An interesting property of the model is that it exhibits a phase transition at finite temperature to a Bose-Einstein condensate (BEC) of hard core bosons [10, 12]. This can be inferred directly from the free energy per site $F / L=\Lambda_{\min } / \beta$ where

$$
\begin{gathered}
\Lambda_{\min }=\beta p^{2}+\min _{\mu \in[0, \min (p, q)]}(\beta \mu(1-\mu)+\mu \log \mu+ \\
\quad(1-\mu) \log (1-\mu)),
\end{gathered}
$$

up to corrections of the order $o\left(L^{-1}\right)$. Here and below we denote $\beta=1 / T, \mu=r / L, p=N / L$ and $q=1-p$. The extremum condition for $\Lambda_{\text {min }}$ leads to the equation

$$
\beta^{*}\left(\mu^{*}\right)=\frac{1}{\left(1-2 \mu^{*}\right)} \ln \left(\frac{1-\mu^{*}}{\mu^{*}}\right),
$$

from which we see that for $T>T_{c}=\left(\beta^{*}(p)\right)^{-1}$ there is no solution for $\mu^{*}$ and the minimum of (3) is reached at the end of the interval $\mu=\min (p, q)$. For $T<T_{c}$, the minimum of (3) is inside the interval and is given by the solution of (4). It was shown [10] that this phase transition is actually a Bose-Einstein condensation with the density of particles in the condensate given by

$$
\rho_{c}=\left(p-p^{*}(\beta)\right)\left(q-p^{*}(\beta)\right)
$$

for $T<T_{c}$ and zero otherwise. In the following we shall characterize the behavior of the VNE across this phase transition.

2. Behavior of the VNE across a classical phase transition. At $\mathrm{T}=0$ the entanglement properties of a subsystem of size $n$ with respect to the rest of the system (seen as environment) can be characterized by the VNE

$$
S_{(n)}=-\operatorname{tr}\left(\rho_{(n)} \log _{2} \rho_{(n)}\right)=-\sum \lambda_{k} \log _{2} \lambda_{k},
$$

where $\lambda_{k}$ are the eigenvalues of the reduced density matrix $\rho_{(n)}$, obtained from the density matrix $\rho$ of the whole system as $\rho_{(n)}=t r_{(L-n)} \rho$. Since we are interested in the behavior of the VNE across the finite temperature BEC phase transition described above, we introduce the thermal von Neumann entropy for a block of size $n$ as follows

$$
S_{(n)}(\beta)=\frac{1}{Z} \sum_{r=0}^{N} d_{r} e^{-\beta E_{r}} \operatorname{Tr}\left(\rho_{(n)}(r) \log _{2} \rho_{(n)}(r)\right),
$$

where $Z$ denotes the partition function. Note that at zero temperature the density matrix of the whole system is a projector on the completely symmetric ground state, $\rho=|\Psi(L, N)\rangle\langle\Psi(L, N)|$ and due to the permutational symmetry, $S_{(n)}$ does not depend on the choice of the sites in the block but only on its size $n$. The corresponding VNE was obtained in [15] where it was shown that $\lambda_{k}=$ $\left(\begin{array}{l}n \\ k\end{array}\right)\left(\begin{array}{l}L-n \\ N-k\end{array}\right) /\left(\begin{array}{c}L \\ N\end{array}\right)$, where $k=0,1, \ldots \min (n, N)$. In the limit of large $n$ von Neumann entropy becomes

$$
S_{(n)} \approx \frac{1}{2} \log _{2}(2 \pi e p q)+\frac{1}{2} \log _{2} \frac{n(L-n)}{L} .
$$

To generalize (8) to arbitrary temperatures we remark that the reduced density matrix for temperatures $T>T_{c}$, $L \rightarrow \infty$ is

$$
\rho_{(n)}=\left.\frac{1}{Z} \sum_{r=0}^{N} d_{r} e^{-\beta E_{r}} \rho_{(n)}(r) \approx \rho_{(n)}\right|_{\mu=\min (p, q)},
$$

while for $T<T_{c}$ we have $\rho_{(n)}=\rho_{(n)}\left(\mu^{*}\right)$ with $\mu^{*}$ given by (4). Thus, to compute the temperature-dependent von Neumann entropy we need to know the eigenvalues of the reduced density matrix $\rho_{(n)}$ for arbitrary YT states. The following theorem provides the result.

3. Eigenvalues of $\rho_{(n)}$ and general properties.

Theorem. The eigenvalues of the reduced density matrix $\rho_{(n)}$ of the eigenstates of $H$ with $N$ particles belonging to the irreps of $S_{L}$ characterized by YTs of type $\{L-r, r\}$, with $r<\min (N,[L / 2])$ are

$$
\begin{aligned}
& \lambda(L, N, n, r, k, s)=\frac{\left(\begin{array}{c}
L-n \\
N-k
\end{array}\right)}{\left(\begin{array}{c}
L \\
N
\end{array}\right)} \sum_{i=0}^{k-s}\left(\begin{array}{c}
k-s \\
i
\end{array}\right)\left(\begin{array}{c}
n-k-s \\
i
\end{array}\right) \times \\
& \sum_{j=0}^{k-i}(-1)^{j} \frac{\left(\begin{array}{c}
s \\
j
\end{array}\right)}{\left(\begin{array}{c}
L-N \\
j+i
\end{array}\right)\left(\begin{array}{c}
N \\
j+i
\end{array}\right)} \sum_{m=0}^{j+i}(-1)^{m}\left(\begin{array}{c}
L-N-r \\
j+i-m
\end{array}\right)\left(\begin{array}{c}
N-r \\
j+i-m
\end{array}\right)\left(\begin{array}{c}
r \\
m
\end{array}\right)
\end{aligned}
$$

with $k, s$, quantum numbers assuming the values $k=$ $0,1, \ldots, n$, and $s=0,1, \ldots, \min (k, n-k)$. The corresponding degeneracies coincide with the dimension of a YTs of type $\{n, s\}$, i.e. $\operatorname{deg} \lambda(L, N, n, r, k, s)=\left(\begin{array}{c}n \\ s\end{array}\right)-\left(\begin{array}{c}n \\ s-1\end{array}\right)$.

The theorem follows from the block diagonalization of the reduced density matrix with respect to the number of particles and to the irreps of $S_{n}$. More precisely, $\rho_{(n)}$ can be block diagonalized with respect to the number of bosons $k$ appearing in the block, this leading to $n+1$ diagonal blocks $B_{k}, k=0,1, \ldots, n$. Each $B_{k}$ can be further diagonalized with respect of the irreps of $S_{n}$ which are compatible with that value of $k$, this leading to $k+1$ blocks associated to the YT of type $\{n-s, s\}$ with $s=0, \ldots, \min (k, n-k)$. Notice that the above decomposition implies that the block $B_{k}$ has dimension $\sum_{i=0}^{k}\left(\left(\begin{array}{l}n \\ i\end{array}\right)-\left(\begin{array}{l}n \\ i-1\end{array}\right)\right)=\left(\begin{array}{l}n \\ k\end{array}\right)$ and the dimension of the ma$\operatorname{trix} \rho_{(n)}$ is $\sum_{k=0}^{n}\left(\begin{array}{l}n \\ k\end{array}\right)=2^{n}$, as it should be. This also clarifies the meaning of the quantum numbers $k, s$, and explains the degeneracies given in the theorem. A full proof of the theorem will be given elsewhere.

Before using the theorem we shall give some general properties of the eigenvalues (10) and consider some limiting cases from which the correctness of the result (10) can be inferred. 
a) One can check by direct inspection that, as consequence of the above block diagonalization, the eigenvalues in (10) satisfy the following remarkable sums with respect to $s$ and $k$

$$
\begin{aligned}
& \sum_{s=0}^{k} \lambda_{k s} \operatorname{deg}\left(\lambda_{k s}\right)=\frac{\left(\begin{array}{l}
L-n \\
N-k
\end{array}\right)\left(\begin{array}{l}
n \\
k
\end{array}\right)}{\left(\begin{array}{l}
L \\
N
\end{array}\right)}, \\
& \sum_{k=s}^{n-s} \lambda_{k s}=\frac{\left(\begin{array}{c}
L-n \\
r-s
\end{array}\right)-\left(\begin{array}{l}
L-n \\
r+s-n-1
\end{array}\right)}{\left(\begin{array}{l}
L \\
r
\end{array}\right)-\left(\begin{array}{l}
L \\
r-1
\end{array}\right)},
\end{aligned}
$$

where hereafter we use $\lambda_{k s}$ as a shorthand notation for $\lambda(L, N, n, r, k, s)$. One can easily verify that the above sums both lead to the correct normalization of $\rho_{(n)}$ : $\operatorname{tr} \rho_{(n)}=1$. Indeed, by using Eq.(11), we have

$$
\operatorname{tr} \rho_{n}=\sum_{k=0}^{n} \sum_{s=0}^{\min (k, n-k)} \lambda_{k, s} \operatorname{deg}\left(\lambda_{k s}\right)=\sum_{k=0}^{n} \frac{\left(\begin{array}{c}
L-n \\
N-k
\end{array}\right)\left(\begin{array}{l}
n \\
k
\end{array}\right)}{\left(\begin{array}{l}
L \\
N
\end{array}\right)}=1 .
$$

A similar expression is obtained by interchanging the order of the sums and using Eq. (12).

b) Case $r=0$. In this case in the sum over $m$ in (10) only the term $m=0$ survives and, since $r=0$ implies $s=0$, also the sum over $j$ has only one term $j=0$. The eigenvalues (10) then reduce to

$$
\lambda_{k 0}=\frac{\left(\begin{array}{c}
L-n \\
N-k
\end{array}\right)}{\left(\begin{array}{c}
L \\
N
\end{array}\right)} \sum_{i=0}^{k}\left(\begin{array}{c}
k \\
i
\end{array}\right)\left(\begin{array}{c}
n-k \\
i
\end{array}\right)=\frac{\left(\begin{array}{c}
L-n \\
N-k
\end{array}\right)}{\left(\begin{array}{l}
L \\
N
\end{array}\right)}\left(\begin{array}{l}
n \\
k
\end{array}\right),
$$

reproducing the exact result obtained for the completely symmetric ground states in [15].

Although explicit (i.e. summed) expressions for the eigenvalues can be derived also for some other particular case (to be reported elsewhere), a summed expression of Eq. (10) seems to be unlikely in the general case.

In the following we will work in the thermodynamic limit $L \rightarrow \infty$ for which the expression for eigenvalues simplifies drastically. Recalling that $\lim _{L \rightarrow \infty}(r / L)=\mu$, $\lim _{L \rightarrow \infty}(N / L)=p$, we obtain

$$
\begin{aligned}
& \lim _{L \rightarrow \infty} \frac{\left(\begin{array}{c}
L-n \\
N-k
\end{array}\right)}{\left(\begin{array}{l}
L \\
N
\end{array}\right)}=p^{n-k} q^{k}, \\
& \lim _{L \rightarrow \infty} \sum_{m=0}^{j}(-1)^{m} \frac{\left(\begin{array}{c}
N-r \\
j-m
\end{array}\right)\left(\begin{array}{c}
L-N-r \\
j-m
\end{array}\right)\left(\begin{array}{c}
r \\
m
\end{array}\right)}{\left(\begin{array}{c}
N \\
j
\end{array}\right)\left(\begin{array}{c}
L-N \\
j
\end{array}\right)}=\eta^{j},
\end{aligned}
$$

where

$$
\eta=\frac{(p-\mu)(q-\mu)}{p q}
$$

plays the role of the order parameter $0 \leq \eta \leq 1$, with $\eta=0$ for $T \geq T_{c}$ and $\eta>0$ for $T \leq T_{c}$. Inserting these expressions into (10) and carrying out the summation $\sum_{j=i}^{k} \eta^{j}(-1)^{j}\left({ }_{j-i}^{s}\right)=(-\eta)^{i}(1-\eta)^{s}$ we obtain

$$
\begin{gathered}
\lim _{L \rightarrow \infty} \lambda_{k s}=p^{n-k} q^{k}(1-\eta)^{s} \sum_{i=0}^{k-s} \eta^{i}\left(\begin{array}{c}
k-s \\
i
\end{array}\right)\left(\begin{array}{c}
n-k-s \\
i
\end{array}\right)= \\
=p^{n-k} q^{k}(1-\eta)_{2}^{s} F_{1}(-k+s, k-n+s ; 1 ; \eta),
\end{gathered}
$$
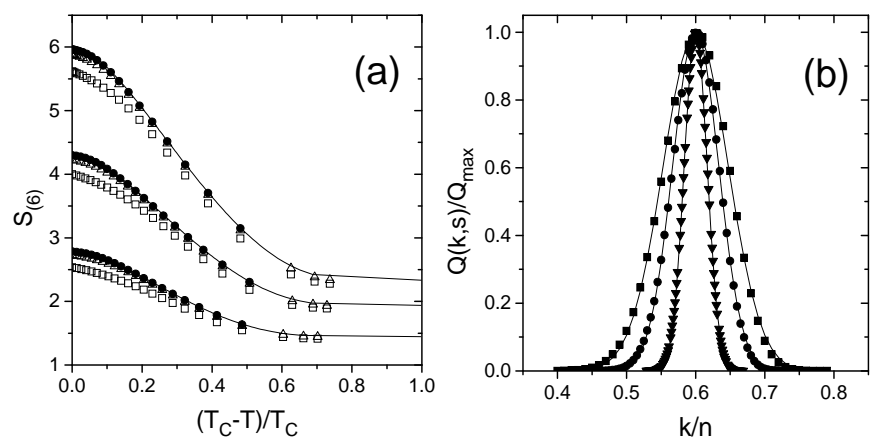

Figure 1: (a) Von Neumann entropy versus temperature for $n=6$, obtained from Eq.(7) for different system sizes: $L=50,200,700$ (squares, triangles and circles respectively). Continuous curves denote the corresponding quantity in the thermodynamic limit (17) while different groups of curves correspond to different densities of hardcore bosons in the system $N / L=0.1,0.2,0.5$ (from down up). (b) Quantity $Q(k, s) / Q_{\max }=\lambda_{k s} \operatorname{deg}\left(\lambda_{k s}\right) / Q_{\max }$ computed from (17) for fixed $s$, versus $k / n$, for $n=100,200,800$ (squares, circles and triangles, respectively). Parameters are $p=0.4$, $\mu=0.2=s / n$. Curves are given by the analytic prediction (19).

where ${ }_{2} F_{1}(a, b ; c ; d)$ is Gauss hypergeometric function. In Fig[1(a) we compare the von Neumann entropy in (7) with the thermodynamic limit (17), for different temperatures and various particle densities.

4. Classical and quantum parts of the von Neumann entropy. Various limits of the above formula are discussed below.

i) Zero temperature limit $T \rightarrow 0$. In this case $\eta=1$, and from (17) we have $\lambda_{k 0}=p^{n-k} q^{k}\left(\begin{array}{l}n \\ k\end{array}\right)$. This is just the limit $L \rightarrow \infty$ of Eq. (13), reproducing the results obtained in [15].

ii) High temperatures $T \geq T_{c}$. In this case $\eta=0$ and the eigenvalues (17) become $s$-independent $\lambda_{k s}=p^{n-k} q^{k}$. This leads to the extensive classical entropy (Gibbs entropy) of an ideal gas with excluded volume

$$
S_{(n)}\left(T \geq T_{c}\right)=-n(p \log p+(1-p) \log (1-p)) .
$$

iii) Intermediate temperatures $0<T<T_{c}$. This case $0<\eta<1$ is the most interesting one since the VNE has both classical (due to degeneracy) and quantum (due to entanglement) contributions. Analyzing the sum in (17) one can show that the variable $Q(k, s)=\lim _{L \rightarrow \infty} \lambda_{k s} \operatorname{deg}\left(\lambda_{k s}\right)$ is Gaussian-distributed with the mean $\langle k / n\rangle=q,\langle s / n\rangle=\mu$ :

$$
Q(k, s) \approx Q_{\max } e^{-\left[\frac{(s-n \mu)^{2}}{2 C}+\frac{(k-n q)^{2}}{2 D}+\frac{(s-n \mu)(k-n q)}{B}\right]},
$$

with $Q_{\max }=(1-2 \mu)(2 \pi n \sqrt{\mu(1-\mu)(p-\mu)(q-\mu)})$, and

$$
\begin{aligned}
C^{-1} & =\frac{1}{n \mu(1-\mu)}+\frac{(p-q)^{2}}{n(p-\mu)(q-\mu)} \\
D^{-1} & =\frac{(1-2 \mu)^{2}}{n(p-\mu)(q-\mu)}, B^{-1}=\frac{(1-2 \mu)(p-q)}{n(p-\mu)(q-\mu)} .
\end{aligned}
$$



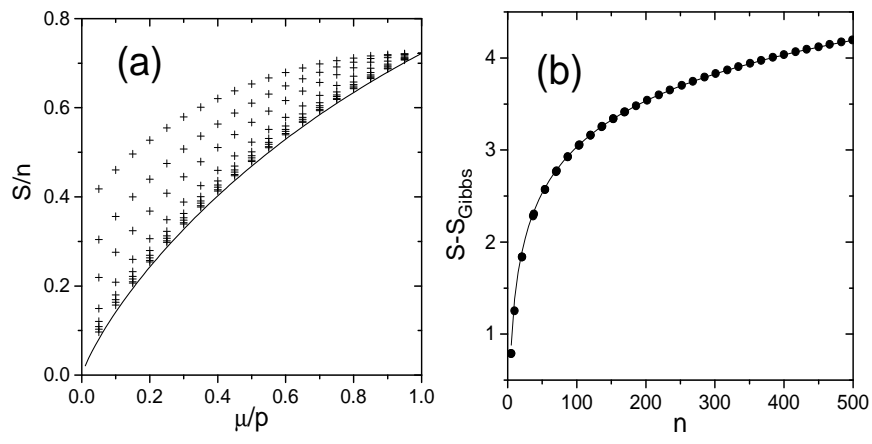

Figure 2: (a). Extensive part of the von- Neumann entropy of a block $\lim _{n \rightarrow \infty} S_{(n)} / n$ versus renormalized temperature $\mu^{*} / p$ for $p=0.2$. Comparison of analytic prediction (23) (curve) with the exact calculations from (17) for finite $n=$ $5,10,50,100,150,200,300,400$ (points approaching the curve from above). (b). "Quantum" part of the von Neumann entropy $S(n)-S_{\text {Gibbs }}(n)$ versus block-size $n$, for $p=0.3$ and $\mu^{*}=0.18$ (points). The continuous curve refers to the fitting function $\frac{1}{2} \log _{2} n-0.289$.

In Fig:1(b) we compare the distribution of the eigenvalues obtained from direct calculations with the Gaussian distribution (19), from which we see that the agreement is excellent. Using the above expression of $Q(k, s)$ we calculate the VNE as

$$
S_{(n)}=-\sum_{k=0}^{n} \sum_{s=0}^{\min (k, n-k)} Q(k, s) \log _{2} \frac{Q(k, s)}{\operatorname{deg} \lambda_{k s}} .
$$

Substituting the sums with the integrals and using the normalization $\sum Q(k, s)=1$ we obtain

$S_{(n)} \approx-n\left(\mu \log _{2} \mu-(1-\mu) \log _{2}(1-\mu)\right)+\frac{1}{2} \log _{2} n+R(q, \mu)$

where $R(q, \mu)$ is a non-universal $n$-independent constant. The first term at the right-hand side of (21) gives the major contribution in the large $n$ limit, and coincides with the Gibbs entropy obtained from the spectrum of the whole system (2) in the thermodynamic limit $E(L, \mu, p) / L \approx \mu-\mu^{2}+p^{2}, d_{r} \approx \frac{1-2 \mu}{(1-\mu)^{L-r+1} \mu^{r}}$, as

$$
\frac{S_{G i b b s}}{L}=\lim _{L \rightarrow \infty} \frac{\beta\langle E-F\rangle}{L},
$$

where $F / L=\Lambda_{\min } / \beta$ and $\Lambda_{\min }$ is given by (3). Inserting the expressions for $E$ and $F$ into (22), we obtain

$$
S_{\text {Gibbs }} / L=-\mu^{*} \log \mu^{*}-\left(1-\mu^{*}\right) \log \left(1-\mu^{*}\right)
$$

where $\mu^{*}(T)$ is defined in Eq.(4). The term $\frac{1}{2} \log _{2} n$ in (21) coincides, up to a constant, with the entanglement entropy at zero temperature (8) and can therefore be interpreted as entanglement entropy part in the von Neumann entropy at finite temperatures, defined as

$$
S_{\text {ent }}(n, T)=S_{(n)}-n \lim _{L \rightarrow \infty} \frac{E-F}{T L}=\frac{1}{2} \log _{2} n+R(q, \mu) .
$$

Note that this contribution disappears at $T_{c}$ and above. In Fig 2 the extensive (panel (a)) and "quantum" parts (panel(b)) of the VNE are depicted.

5 Conclusions. We have calculated the spectrum of the reduced density matrix and von Neumann entropy of a block of $n$ sites, for a quantum model with permutational symmetry, as function of temperature and particle density. It is shown that eigenvalues of the reduced density matrix in the thermodynamic limit are parametrized by the single parameter defined in (16), which also turns out to be the order parameter of the problem. We defined the entropy of entanglement for finite temperature as the difference between the VNE and its extensive part (coinciding with the Gibbs entropy), and demonstrated its disappearance above the classical phase transition. For all temperatures below the critical one, the entropy of entanglement scales as $\frac{1}{2} \log n$. We expect this results to be valid also for other quantum systems with permutational symmetry (mean field models) exhibiting finite temperature phase transitions.

Acknowledgements. V.P. thanks the Department of Physics of the University of Salerno for a research grant (Assegno di Ricerca n.1013-2006) during which this work was done.

[1] M. A. Nielsen, I.L. Chuang, Quantum computation and quantum information, Cambridge University Press, 2000.

[2] G. Benenti, G. Casati, G. Strini, Principles of quantum computation and information, Vol II: Basic Tools and special Topics, World Scientific, Singapore, 2007.

[3] V.Vedral, M.B. Plenio, M.A. Rippin and P.L. Knight, Phys. Rev. Lett. 78 (1997) 2275.

[4] V. Vedral, Rev. Mod. Phys. 74, 1 (2002) 216.

[5] G. Vidal, J. I. Latorre, E. Rico, and A. Kitaev, Phys. Rev. Lett. 90 (2003) 227902.

[6] V. E. Korepin, Phys.Rev.Lett. 92 (2004) 096402.

[7] P. Calabrese, J. Cardy, J. Stat. Mech.: Theor. Exp. (2004) P06002.

[8] H. J. Lipkin, N. Meshkov and A. J. Glick, Nucl. Phys. 62 (1965) 188.

[9] B. Tóth, J. Stat. Phys. 61 (1990) 749.

[10] O. Penrose, J. Stat. Phys. 63 (1991) 761.

[11] G. Albertini, V.E. Korepin and A. Schadschneider, J. Phys. A 28 (1995) L303.

[12] M. Salerno, Phys. Rev. E, 50 (1994) 4528.

[13] J.I. Latorre, R.Orús, E. Rico and J. Vidal Phys. Rev. A, 71064101 (2005).

[14] V. Popkov, M. Salerno and G. Schütz, Phys. Rev. A 72 (2005) 032327.

[15] V. Popkov and M. Salerno, Phys. Rev. A 71 (2005) 012301.

[16] M. Hayashi,D. Markham, M. Murao, M. Owari, and S. Virmani, Phys. Rev. A 77 (2008) 012104.

[17] M. Salerno and J. C. Eilbeck Phys. Rev. A 50 (1994) 553. 